\title{
Global gene expression in organ-specific cold stress response in Arabidopsis thaliana
}

\author{
A. Klepikova',2*, I. Kulakovsky ${ }^{3,4}$, A. Kasianov ${ }^{2,3}$, M. Logacheva ${ }^{2,5,6}$, A. Penin ${ }^{1,2,5}$ \\ ${ }^{1}$ Institute for Information Transmission Problems RAS, Moscow, Russia \\ ${ }^{2}$ Lomonosov Moscow State University, Moscow, Russia \\ ${ }^{3}$ N.I. Vavilov Institute of General Genetics RAS, Moscow, Russia \\ ${ }^{4}$ Engelhard Institute of Molecular Biology RAS, Moscow, Russia \\ ${ }^{5}$ Kazan Federal University, Kazan, Russia \\ ${ }^{6}$ Skolkovo Institute of Science and Technology, Skolkovo, Russia \\ *e-mail: annklepikova@gmail.com
}

Key words: calcium overload, rhythm disturbances, cardiac mechanics

Motivation and Aim: Plants face unavoidable environmental perturbations because of their sessile nature and have evolved various tolerance mechanisms to stress conditions. The process of increasing tolerance to freezing - cold acclimation - is well-studied in Arabidopsis thaliana. The main regulatory genes controlling cold response, such as $C B F$ genes, were identified by classic genetics methods [1] and lots of transcriptomic studies were conducted on cold treated plants [2]. However, most of these papers were focused on whole aerial parts of plants or leaves and there have been no attempts to compare the expression profiles in different parts of plants during cold exposure

Methods and Algorithms: For this study we have chosen six most different biological samples of $A$. thaliana. The samples were collected from plants under normal condition as control and after 3 and 27 hours of cold $\left(+4^{\circ} \mathrm{C}\right)$ treatment. Expression features of cold response in various organs were analyzed.

Results: We have analyzed differential expression between cold-treated and control samples for each organ and different number of DE genes for each sample and 15,459 $\mathrm{DE}$ genes at all. The majority of the genes were not identified as stress-response in Gene Ontology (GO). Upregulated in all samples genes were enriched in stress-related GO terms and were "core" genes for cold resistance. Unique for samples genes were enriched in organ-specific manner. We found specific features of expression patterns in whole plant for organ-specific DE genes, such as activation of pollen-specific genes in leaves under cold treatment. We found new TFs to be involved in cold response.

Conclusion: Our results showed that the gene expression changes were highly organspecific. When only a small number of cold-response genes were common in all samples acting as "core", there were a great variety of organ-specific genes with distinct expression specificities. Thus, while the mechanisms of cold stress response are common in all plants, in every organ they are modified in a unique fashion, including the recruitment of genes that are expressed in other organs in non-stress conditions. The results are summarized in the TraVa public database.

Acknowledgements: Supported by the RSF (project No. 14-50-00150).

\section{References}

1. Gilmour S.J. et al. (1998) Low temperature regulation of the Arabidopsis CBF family of AP2 transcriptional activators as an early step in cold-induced COR gene expression. Plant J. 16:433-442.

2. Kilian J. et al. (2007) The AtGenExpress global stress expression data set: protocols, evaluation and model data analysis of UV-B light, drought and cold stress responses. Plant J. 50:347-363. 